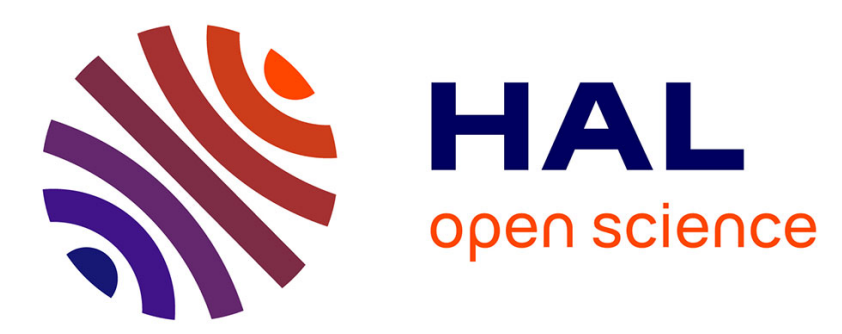

\title{
Study of the Effect of the Directness of the Interaction on Novice Users When Drawing Straight Lines
}

Élisabeth Rousset, François Bérard, Michaël Ortega

\section{To cite this version:}

Élisabeth Rousset, François Bérard, Michaël Ortega. Study of the Effect of the Directness of the Interaction on Novice Users When Drawing Straight Lines. 27ème conférence francophone sur l'Interaction Homme-Machine., Oct 2015, Toulouse, France. pp.a14, 10.1145/2820619.2820633 . hal-01218668

\section{HAL Id: hal-01218668 \\ https://hal.science/hal-01218668}

Submitted on 21 Oct 2015

HAL is a multi-disciplinary open access archive for the deposit and dissemination of scientific research documents, whether they are published or not. The documents may come from teaching and research institutions in France or abroad, or from public or private research centers.
L'archive ouverte pluridisciplinaire HAL, est destinée au dépôt et à la diffusion de documents scientifiques de niveau recherche, publiés ou non, émanant des établissements d'enseignement et de recherche français ou étrangers, des laboratoires publics ou privés. 


\title{
Study of the Effect of the Directness of the Interaction on Novice Users When Drawing Straight Lines
}

\author{
Élisabeth Rousset, François Bérard, Michaël Ortega \\ Univ. Grenoble Alpes, LIG, F-38000 Grenoble, France \\ CNRS, LIG, F-38000 Grenoble, France \\ first.last@imag.fr
}

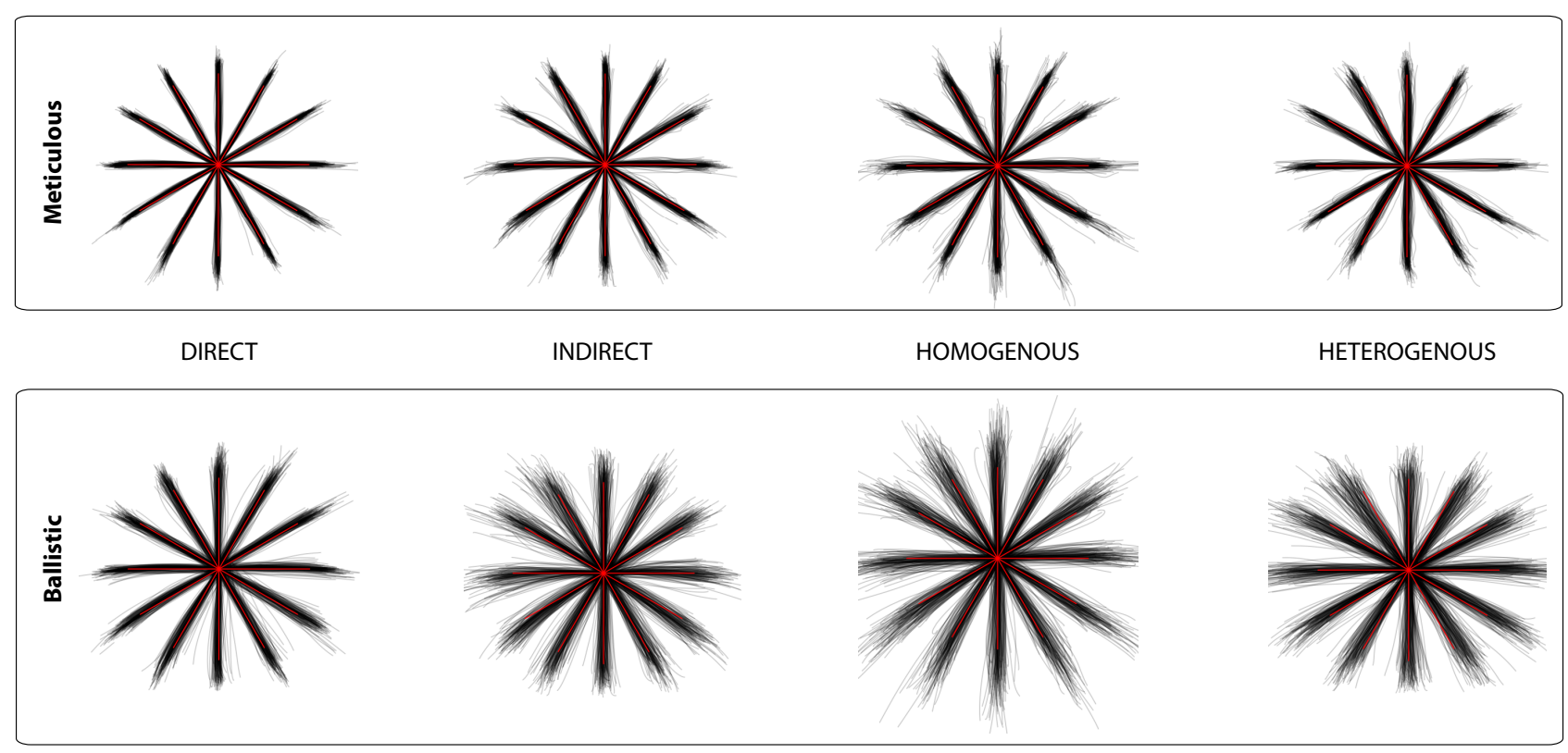

Figure 1. Lines drawn by the participants in the DIRECT, INDIRECT, HoMOGENOUS and HETEROGENOUS conditions, for both meticulous and ballistic drawing. We observe greater irregularities and angle sectors with indirect techniques than with DIRECT in each drawing style, which means worse drawing quality.

\section{ABSTRACT}

Pen-enabled devices are widely used in computer graphics. Some artists use direct devices (interactive pen displays) whereas others use indirect devices (graphics tablets) and this applies to both professionals and hobbyists. In this paper we studied how indirect pen-enabled devices affect drawing performance compared to their direct counter-parts for novice users. We also studied the influence of gain for indirect pen-enabled devices. We considered no gain (i.e. $\mathrm{C} / \mathrm{D}=1$ ), a homogenous gain (gain $=2$ on both the $\mathrm{x}$ and $\mathrm{y}$ axes) and a heterogenous gain (gain $=2$ on the $\mathrm{x}$ axis and gain $=1$ on the $\mathrm{y}$ axis). Results indicate that drawing performance and quality decreased with indirectness and with both homogeneous and heterogeneous gains. In addition, we conducted a qualitative study showing that participants preferred direct devices.

(c) ACM , 2015. This is the author's version of the work. It is posted here by permission of ACM for your personal use. Not for redistribution. The definitive version was published in Actes de la 27ème conférence francophone sur I'Interaction Homme-M achine, 2015. http://dx.doi.org/10.1145/2820619.2820633

\section{Key Words}

Graphic arts and interaction; Evaluation and metrics.

\section{ACM Classification Keywords}

H.5.2 User Interfaces: Input devices and strategies.

\section{INTRODUCTION}

Digital pens are widely used in computer graphics for the execution of various tasks: from simple 2D illustration to $3 \mathrm{D}$ painting, modeling or animating. By mimicking physical pens or brushes, digital pens are suitable for painting and drawing: they offer a grip that allows efficient and accurate control of the nib's position, leaning and pressure. Digital pens are used in two forms of interaction: direct and indirect. Interactive pen displays support a direct interaction in which the virtual ink is laid at the nib's position, much like with physical pens on paper (e.g. Figure 2, left). Graphics tablets offer an indirect interaction where the nib's position and the ink are dissociated: the pen is typically used on a horizontal tablet and controls a pointer displayed on a vertical screen (e.g. Figure 2, right). Both forms of interactions have benefits and drawbacks.

Direct pens appear as being more similar to physical pens and paper than indirect pens. As such, users' skills ac- 


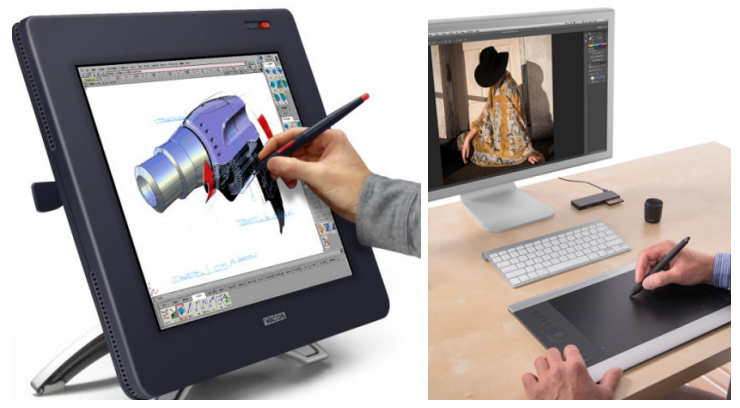

Figure 2. Interactive pen display (direct interaction) vs. Graphics tablet (indirect interaction)

quired in the physical world may transfer better to direct pens compared to indirect pens, much in the same way that physical skills transfer better to direct pointing compared to indirect pointing [4]. However, direct interaction suffers from the parallax resulting from the display thickness, and from the partial occlusion of the display by the user's arm and hand. In addition, the motor and visual spaces are superposed, which imply that the two spaces can not be both set in their most comfortable orientation: preferably, the motor space should be horizontal so that the hand can rest on the surface, while the visual space should be vertical in order to reduce the neck's strain. Moreover latency perception has lower thresholds in direct input than in indirect input [6] which implies a greater tolerance of users to latency in indirect devices.

Indirect interaction enables the ideal orientation of both motor and display spaces, it suppresses the problems of parallax and occlusion, and it allows the use of a Control/Display (CD) gain that reduces the demand in physical displacements of the pen. However, this results in a complex transformation between the motor and visual spaces. Comprehending this transformation can be difficult for users and may hinder their ability to perform the task. Indeed, comments from both novice and expert users indicate that indirect digital pens are more difficult to handle than direct pens, and that the difficulty results in lower drawing quality. A major manufacturer of both direct and indirect digital pens offers users to train on eye-hand coordination in order to reduce the problem ${ }^{1}$. However, formal studies are required to better understand the implications of using either direct or indirect digital pen interaction, especially for novice/hobbyist users since they have far less practice and training than professionals. While previous research efforts studied the differences between direct and indirect interaction $[12,16]$, direct and indirect pens [7] and the effect of gain distortion [8], these studies focused on pointing performances. The present work studies drawing performance and quality.

When pointing at targets, the pen's trajectory can be adjusted until it reaches the target. This is not the case when drawing since each adjustment will be visible on the resulting stroke. As a consequence, drawing requires that the user moves the cursor in the right direction at any time. Drawing requires that the user is always aware of (1) the

\footnotetext{
${ }^{1}$ See "Eye-hand coordination exercise" in http://www. wacom.eu/_bib_user/dealer/man_onebywacom_en.pdf
}

cursor's position and (2) the direction of the cursor's motion. In the case of direct interaction, (1) is provided by the pen's nib position while (2) corresponds to the motion intended by the user (since display and motor space are in the same frame of reference). In the case of indirect interaction, (1) is supplied by cursor display while hovering over the tablet and (2) is not available (since display and motor space are not in the same frame of reference), (2) can only be "approximatively" inferred from previous positions of the cursor. Our hypothesis is that the bad inference of (2) in indirect drawing leads to more adjustments and to a lower quality of the trajectory compared to direct drawing. This hypothesis is coherent with the experiment from Forlines et al., which showed that pen crossing selection was less efficient in indirect interaction than in direct interaction [7].

In this paper we present a study comparing the effect of "directness" (i.e. either direct or indirect interaction) on novice users' drawing performance and production quality. In particular, we study a basic building block of the more general drawing task: drawing oriented straight lines, and we focus on the orientation of the lines. As a secondary factor, we also study the effect of the gain (i.e. no gain, homogeneous or heterogeneous) when using indirect interaction. The heterogeneous condition was introduced because many graphics tablets with a $4 / 3$ width/height ratio are still used with current computer monitors that have $16 / 10$ or $16 / 9$ ratios.

The next section presents the related work on eye-hand coordination and the quality of drawing productions. We then detail our experiment, discuss its results and conclude.

\section{RELATED WORK}

\section{Eye-hand coordination}

Studies on the eye-hand coordination have been published in neuroscience literature. In particular, these studies inform about the effect of the lack of coordination resulting from the eye-hand dissociation in indirect drawing.

Vercher and al. showed that the arm proprioception has an important role in the calibration of the temporal relationship between the arm and eye movements. They hypothesize that dissociation may lead to a bad synchronization between that eyes and the hand because of different frames of reference [19] which is the case in indirect interaction.

Ketcham et al. found a correlation between eye and hand movement: they studied how they were related during complex gestures and showed that the eyes were locked around few locations of the hand trajectory, which correspond to curvature maxima [11]. The eyes precede the hand during the movement, and saccades occur when the hand reaches minimum speed. More recently Gowen and al. studied the eyes' movements during a tracing task (e.g. re-drawing directly on top of a model) and a drawing task (eg. drawing without any model) [9]. They showed that when tracing, the eyes are fixed slightly ahead of the pen tip and go forward by small saccades. They refer to this 
behavior as close pursuit since the pen's nib closely follows the trajectory of the eyes. Drawing implies a different behavior referred to as target locking: the eyes' fixation is far ahead from the pen's nib and remains stable until the nib reaches the neighborhood of the fixation. There are fewer but longer fixations in this behaviour and changes are made with large saccades. As we are interested in drawing tasks (i.e. not tracing), our study focuses on the effect of the directness of the interaction on the target locking behavior.

Tchalenko's studied eye movements when drawing simple lines [18]. He showed that the target locking behavior was induced when participants had to draw a straight line from a displayed origin to a non-displayed target. The location of the target was guessed by the participant according to surrounding elements in the canvas, such as the intersection of the prolongations of two segments. We defined our experimental task following Tchalenko's, and in particular we did not display the actual target of the drawing task. Tchalenko did not find any difference between the behavior of novice and professional designers. In addition, he found that the eyes' movement strategy was the same for straight lines and curves. This last finding was confirmed by Reina and al who found that the eyes's movement during curve drawing was an iteration of target lockings at the location of maximum curvature [15]. These results indicate that our study on drawing straight lines might generalize to curved lines, although further experiments will be required to provide a definite answer.

\section{Trajectory metrics}

Accot et al. proposed an extension of Fitts' Law for tasks involving cursor trajectories within "tunnels" [1]. However, they compare the tunnels, and not the lines intended by the user. Other efforts proposed curve matching distances, such as Fréchet's [2] and Hausdorff's [3], or customized distances $[14,17]$. These metrics depend on both the position and the orientation of the trajectory, and the orientation cannot be isolated. However, isolating the orientation is crucial for our study because measures based on position introduce supplemental shift information.

Mackenzie et al. introduced six measures of a trajectory's quality [13]. Four are only based on position, and two on orientation: Mouvement Direction Change (MDC) and Orthogonal Direction Change $(O D C)$. As the second measure is not relevant in our context, we use a generalisation of the MDC, that takes into account the inflection points.

Keefe and al. proposed a measure of the direction difference between curves [10]. We used a simplified version for straight lines in our experiment.

\section{EXPERIMENT}

\section{Apparatus}

The experiment was performed on a laptop computer with an Intel Core i7, $2.6 \mathrm{GHz}$ processor and a NVidia GT $650 \mathrm{M}$ graphic card, using a custom-designed application programmed in the Cocoa framework. We used a 24 inch standard display at its native resolution of $1920 \times 1200$ pixels, and a 24 inch interactive pen display (Wacom Cintiq

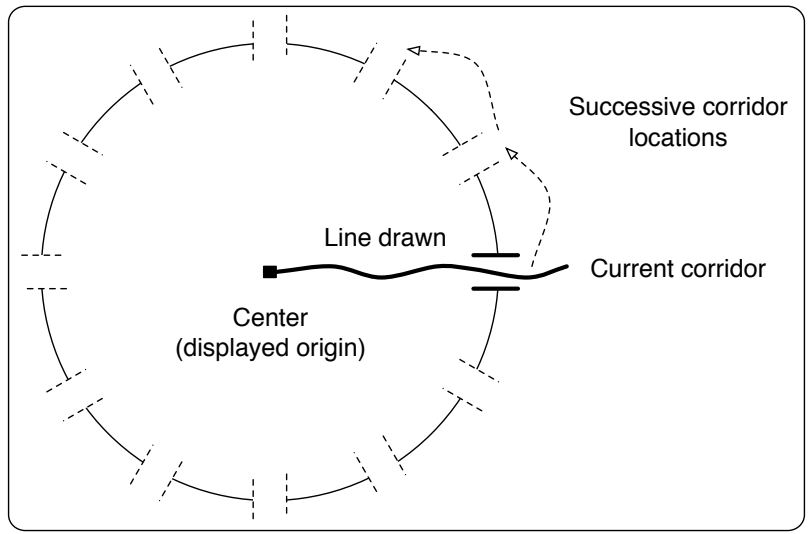

Figure 3. The task: participants have to draw a straight radius from the center of the circle through a corridor. The corridor is successively displayed at twelve different locations on the circle, in a counterclockwise order.

24HD touch, with touch disabled) with the same resolution. Participants used the pen (Wacom Grip Pen) on the pen display both in the direct and the indirect conditions. The strokes generated by the participants were shown on the pen display in the direct condition, and on the standard display in the indirect condition (Figure 4). The pressure sensitivity of the pen was disabled during the experiments.

\section{Task and Participants}

We chose a task that induced a target locking behaviour since this was identified as the natural behaviour when drawing with physical pen and paper [9]. According to Tchalenko, target locking can be induced by asking participants to draw a line from a displayed origin to a nondisplayed target [18]. Our participants were presented with a circle with $12 \mathrm{~cm}$ radius the center of which was displayed as a small square (the displayed origin). They had to draw a radius from that center to the non-displayed intersection of the middle of a corridor with the circle (Figure 3). The corridor was successively presented at twelve different locations counter-clockwise (Figure 3). We chose to display corridors that were parallel to the radius in order to encourage the drawing of straight lines. A different corridor orientation may affect the trajectory performed by the participants [5].

We asked participants to perform the task in two different drawing styles:

- in meticulous drawing, participants were instructed to carefully and precisely draw straight lines, and to ignore any time constraint;

- in ballistic drawing, participants were instructed to draw lines with a quick movement. This particular gesture is typical of sketching tasks.

We recruited 16 right-handed participants, aged 41 on average ([24-65], stddev=15.4). All were novices in digital drawing.

\section{Interaction techniques}

The tasks were performed in 4 different techniques: 


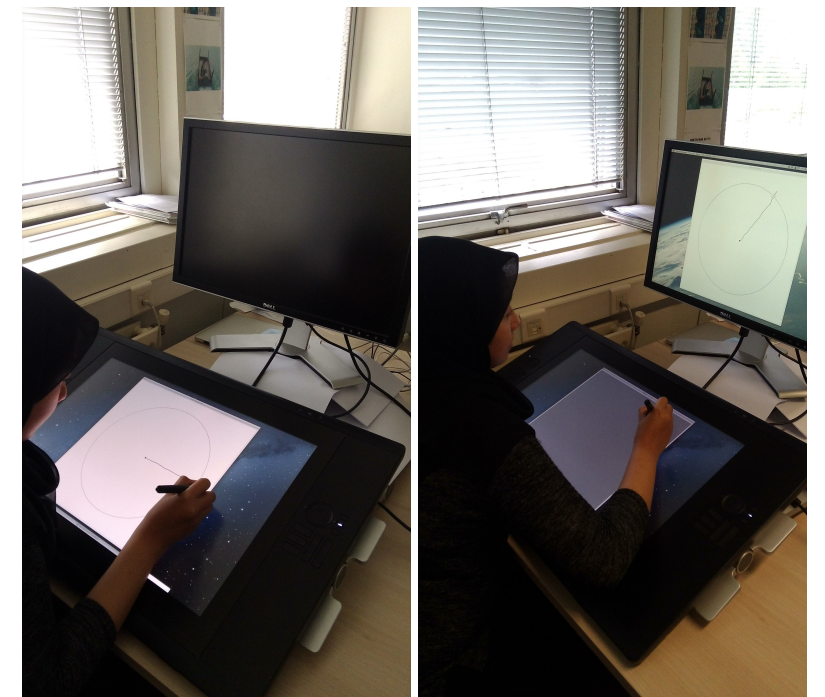

Figure 4. Direct vs. Indirect setup: in direct interaction (left) participants only interact with the pen display. In indirect interaction (right) they draw on the pen display while looking at the standard display

\section{DIRECT}

The circle is shown on the interactive pen display. Participants interact directly on the display (Figure 4, left).

\section{INDIRECT}

The circle is displayed on the standard display. Then the pen is used on the pen display whose output is set to a plain grey area (Figure 4, right). The $\mathrm{C} / \mathrm{D}$ gain is set to 1: the cursor runs the same distance on the screen as the physical displacement of the pen. This is a deviceindependant mesure $(\mathrm{mm} / \mathrm{mm})$.

\section{HOMOGENOUS}

Same as INDIRECT, but using a homogenous gain of 2 . The cursor runs twice the distance on screen as the physical displacement of the pen.

\section{HeTEROGENOUS}

Same as INDIRECT, but using a heterogenous gain: the gain is 2 on the $\mathrm{x}$ axis and 1 on the $\mathrm{y}$ axis.

\section{Design}

The study was conducted in a silent room. Participants were sitting in front of the pen display which was at $30^{\circ}$ of angle from the horizontal (Figure 4). The standard display was placed behind the pen display, aligned with it and facing the participant. At the beginning of a session, the investigator explained the procedure to the participant. We used a within-subject design: each participant drew radii targeting 12 different angles in each of the 4 techniques (DIRECT, InDIRECT, HoMOgENOUS, Heterogenous). Each technique was tested in meticulous and ballistic drawing, with 8 repetitions. Overall, we recorded: 16 (participants) x 4 (technique) $\times 2$ (drawing style) x 12 (angles) x 8 (repetitions) $=12288$ trajectories. Each new combination of technique and drawing style started with a training phase in which participants performed 24 trials ( 2 series of 12 angles). The order of presentation of drawing styles was balanced between
2 groups of 8 participants. Within each groups, we balanced the presentation order of the techniques. In a postexperiment interview, participants scored the techniques according to their preferences.

\section{Measures}

To observe a potential effect of indirectness and gain on drawing performance and quality, we investigated completion time and 3 measures of drawing quality focused on the orientation of the strokes. Completion Time (CT) is the time from the first contact event to the last contact event. The quality of the orientation is evaluated from three measures:

- Orientation Error (OE): the deviation of the global line orientation from the prescription (the straight radius passing through the center of the corridor). This measure is a simplified version of the measure used in Keefe et al. [10]. OE is computed with the following steps, which are illustrated on figure 5. First, we resample the pen events so that no two events are closer than 10 pixels from each other. This results in a succession of small straight segments. We use the median value of the orientation of all the segments as the global orientation of the drawn line. The orientation error $(\mathrm{OE})$ is the absolute difference between this global orientation and the prescription. Compared to a linear regression, this approach is more robust to outliers.

- Orientation Variability (OV): the amount of angle variation during the drawing of the line. This measure uses the sampling from the OE measure. For each segment, we compute its signed deviation compared to the prescription. OV is the absolute difference between the $10 \%$ and $90 \%$ quantiles of the distribution of deviations (figure 5).

- Number of Inflection points (NI): NI is the number of inflection points of the drawn curve.

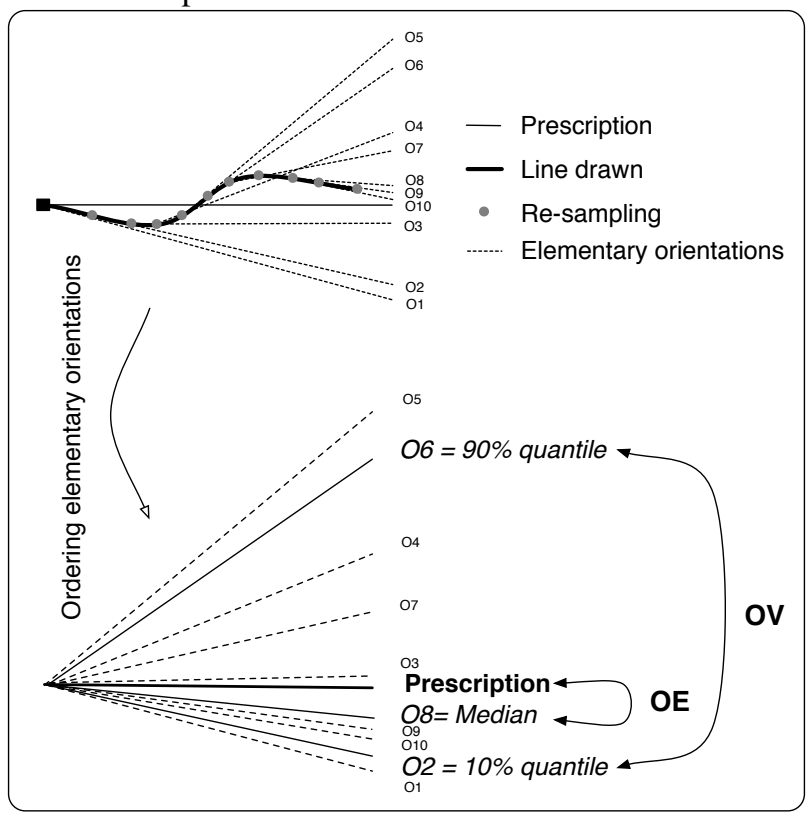

Figure 5. OE and OV computation: After resampling of the line into small segments, the orientations of each segment are ordered. $\mathrm{OE}$ is the absolute difference between the prescription and median orientations. $\mathrm{OV}$ is the absolute difference between the $10 \%$ and $\mathbf{9 0 \%}$ quantile orientations 

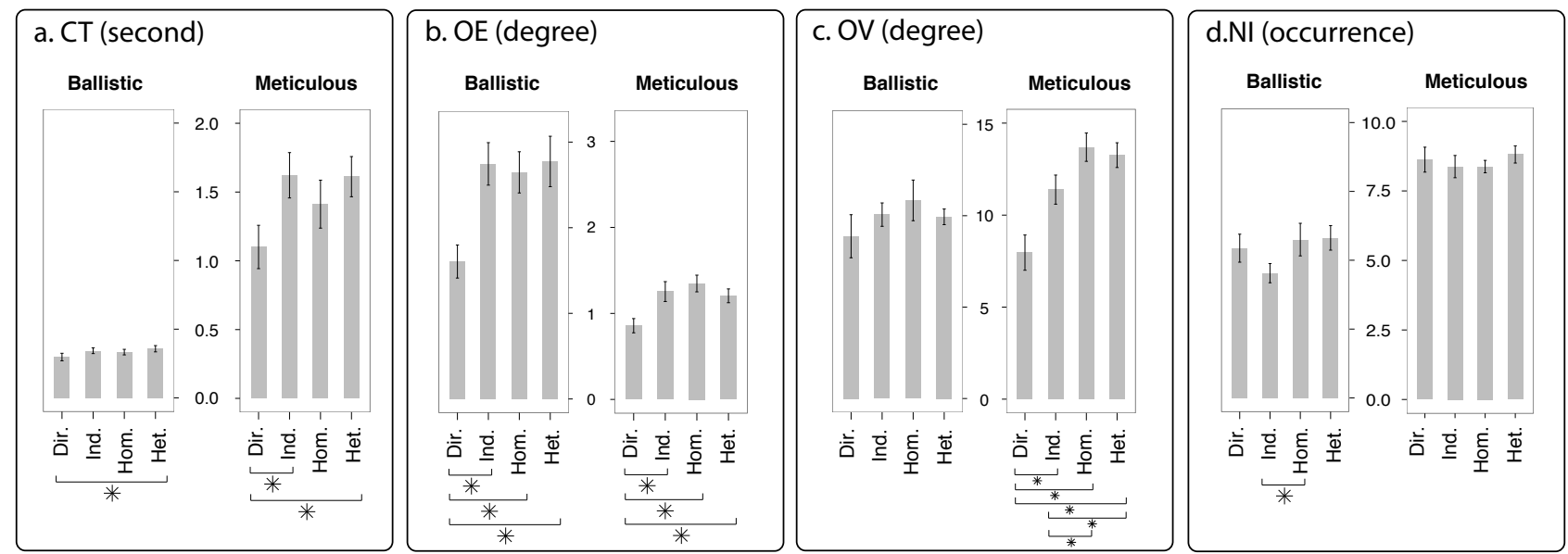

Figure 6. Results (means and 95\% CI): a) Completion Time (CT, in seconds), b) Orientation Error (OE, in degrees), c) Orientation Variability (OV, in degrees) and d) Number of Inflexion points (NI, in occurrences) for the four techniques for both ballistic (left) and meticulous (right) drawing styles

\section{Results}

We first investigated the effect of the angle of the corridor location on OE, OV, NI and CT. Drawing vertical lines, for example, is known to be more difficult than horizontal lines because the corresponding gesture recruits more joints of the arm. However, we did not find any significant effect of the angle on the 4 measures. This could be explained by the fact that we did not constrain participants' posture during the experiment: they could adjust their position to allow the most comfortable gesture in each target orientation. As a consequence, for each measure OE, OV NI and CT and for each combination of participant, drawing type and technique, we aggregated the values for all the angles and we used the median in further computations.

Shapiro tests revealed that the distribution of medians across participants of $\mathrm{OE}, \mathrm{OV}, \mathrm{NI}$ and CT did not follow a Normal distribution. We thus conducted non-parametric statistical tests. Effect of factors were assessed with Friedman tests, and post-hoc pairwise comparison with Wilcoxon tests with Bonferroni adjustment. Results are presented in Figure 6. The superimposition of all the lines drawn by the participants is presented in Figure 1.

\section{Completion Time}

Completion Times (CT) are represented in Figure 6a. A Friedman test revealed a significant global effect of the interaction technique on $\mathrm{CT}$, both in meticulous and ballistic drawing (resp, $\mathrm{p}=1.42 \mathrm{e}-03$ and $\mathrm{p}=5.21 \mathrm{e}-03$ ). Posthocs revealed that (1) for ballistic drawing CT was significantly smaller with DiRECT than with HETEROGENOUS $(p<0.05)$ with a $17 \%$ improvement and (2) in meticulous drawing CT was significantly smaller with DIRECT than with INDIRECT and HeTEROGENOUs $(\mathrm{p}<0.01)$ with a respective improvement of $55 \%$ and $54 \%$. We found no significant difference of CT between the indirect interaction techniques for both ballistic and meticulous drawing.

\section{Orientation Error}

Orientation Errors (OE) are presented in Figure 6b. A Friedman test revealed a significant global effect of the interaction technique on $\mathrm{OE}$, both in meticulous and ballistic drawing (resp. $\mathrm{p}=2.09 \mathrm{e}-05$ and $\mathrm{p}=1.56 \mathrm{e}-05$ ). Posthocs revealed that (1) in ballistic drawing OE was significantly smaller with DIRECT than with INDIRECT, HOMOGENOUS and HETEROGENOUS $(\mathrm{p}<0.001)$ with respective improvements of $43 \%, 38 \%$ and $40 \%$ and (2) in meticulous drawing OE was significantly smaller with DIRECT than with INDIRECT, HOMOGENOUS and HETEROGENOUS $(\mathrm{p}<0.001)$ with respective improvements of $32 \%, 37 \%$, and $29 \%$. We found no significant difference between each one of the indirect techniques both in ballistic and meticulous drawing. This can be observed on Figure 1: the lines drawn with the indirect techniques fit in a larger sector than the ones drawn with DIRECT.

\section{Orientation Variability}

Orientation Variabilities (OV) are presented in Figure 6c. A Friedman test revealed a significant global effect of the interaction technique on $\mathrm{OV}$ in meticulous drawing $(\mathrm{p}=7.22 \mathrm{e}-08)$ but not in ballistic drawing. Post-hocs revealed that in meticulous drawing (1) OV was significantly smaller with DIRECT than with INDIRECT, HOMOGENOUS and HETEROGENOUS $(\mathrm{p}<0.001)$ with respective improvements of $30 \%, 42 \%$ and $38 \%$ and (2) OV was significantly smaller with INDIRECT than with HOMOGENOUS and HETEROGENOUS $(\mathrm{p}<0.05)$ with respective improvements of $17 \%$ and $14 \%$. We found no significant difference between HOMOGENOUS and HETEROGENOUS. This can be observed on the irregularity of the lines in Figure 1: there is more irregularity on the lines drawn with indirect techniques than on the lines drawn with DIRECT.

\section{Number of Inflection points}

Number of Inflection points (NI) are represented in Figure $6 \mathrm{~d}$. A Friedman test revealed a significant global effect of the interaction technique on NI in ballistic drawing $(p=2.63 e-03)$ but not in meticulous drawing. Posthocs revealed that in ballistic drawing NI was significantly smaller with INDIRECT than with HETEROGENOUS ( $\mathrm{p}<$ 0.01 ) with a $25 \%$ improvement. 


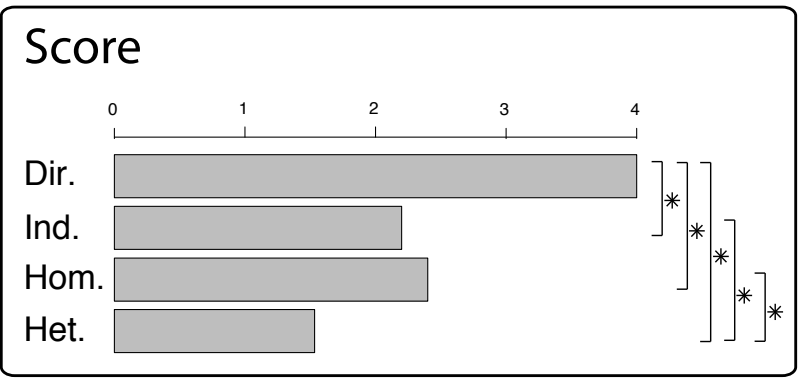

Figure 7. Results: Qualitative score of the techniques (greater is better).

\section{Subjective Evaluation}

Participants' preferences were represented by a score in the set $\{1,2,3,4\}, 4$ being the most preferred technique. Results are presented in Figure 7. A Friedmann rank sum test revealed that there was a significant effect of the interaction technique on participant's rankings $(\mathrm{p}=5.85 \mathrm{e}-07)$. We performed a pairwise comparisons using a Wilcoxon rank sum test with Bonferroni adjustment. It revealed that participants ranked the DIRECT interaction technique significantly better than each one of the indirect techniques $(p<0.001)$. Moreover, we noticed that DIRECT was unanimously ranked as the preferred technique by all participants. The test also revealed that INDIRECT and HoMOGENOUS were significantly ranked above HETEROGENOUS $(\mathrm{p}<0.05)$.

\section{DISCUSSION}

\section{Direct vs. indirect}

Our experiment provides evidence that the direct interaction supported by interactive pen displays is better suited than the indirect interaction of graphics tablets with regard to drawing oriented lines for novice users. Participants were in general faster in the direct interaction technique, while achieving a more accurate general orientation of the stroke and less variation of orientation. From a subjective point of view, participants clearly expressed their preference for the direct interaction, all of them giving it the highest ranking. Some participants felt that they were traveling larger distances in INDIRECT than in DIRECT.

In order to better understand the effect of the eye-hand dissociation in indirect interaction, we focus on the results of the DIRECT and INDIRECT techniques, both using a C/D gain of 1 , and disregard the results of HOMOGENOUS and Heterogenous. This analysis is summarized in table 1.

When performing ballistic drawing, participants had $43 \%$ less orientation error (OE) in DIRECT than in INDIRECT,

\begin{tabular}{|lll|}
\hline & Ballistic & Meticulous \\
\hline CT & - & DIRECT $<$ INDIRECT \\
OE & DIRECT $<$ INDIRECT & DIRECT $<$ INDIRECT \\
OV & - & DIRECT $<$ INDIRECT \\
NI & - & - \\
\hline
\end{tabular}

Table 1. Effect of indirectness on Completion Time (CT), Orientation Error (OE), Orientation Variability $(\mathrm{OV})$ and Number of Inflexion points (NI) for both ballistic (left) and meticulous (right) drawing but we found no significant difference in OV, NI and CT. Our interpretation is as follows: ballistic drawing is constrained in time, which explains the lack of difference in CT. In addition, this leaves fewer opportunities for users to adjust their gesture. Few adjustments implies few NI and few OV, which here again explains the lack of significant differences. With few possible adjustments, the general direction of the stroke is close to the initial direction taken by the hand. Our experiment provides empirical evidences that it is more difficult to throw the hand in the correct orientation when there is eye-hand dissociation, i.e. when using an indirect interaction technique.

When performing meticulous drawing, we found a small but significant difference in OE ( 0.4 degree) between DIRECT and INDIRECT. As the drawing was not constrained in time, participants took all the time needed to draw a "good" line. But to achieve a similar OE, participant required 55\% more time in INDIRECT compared to DIRECT. In addition, even though we found no significant difference in the number of inflection points (NI), DIRECT yielded $30 \%$ less orientation variability (OV) than INDIRECT. One interpretation is that the adjustment frequency is a participant's characteristic, while the adjustment "intensity" depends on the interaction technique. To summarize, participants were able to achieve a stroke orientation almost as good in INDIRECT as in DIRECT when performing meticulous drawing, but at the cost of significantly lower performance and lower production quality. This provides another light to the greater difficulty of drawing oriented lines in indirect interaction compared to direct interaction.

\section{Effect of the gain in indirect techniques}

We now discuss the effect of the gain by focusing on the comparisons between the indirect techniques only (i.e. ignoring the results for DIRECT). Some participants expressed that they appreciated HoMOGENOUS more than INDIRECT because they found it practical to travel less distance on the tablet than needed on the screen. However, other participants complained that it was "too sensitive". Heterogenous was considered "disturbing" by a majority of participants, even though they could not explain why.

The analysis of the quantitative results for indirect interactions is summarized in Table 2. The gain affected the variability of the orientation (OV) in the case of meticulous drawing, with INDIRECT yielding $17 \%$ less variability than Homogenous and $14 \%$ less variability than HeTEROGENOUS. Furthermore, INDIRECT lead to $25 \%$ less

\begin{tabular}{lll|}
\hline & Ballistic & Meticulous \\
\hline CT & - & - \\
OE & - & - \\
OV & - & INDIRECT $<$ HOMOGENOUS \\
& & INDIRECT $<$ HETEROGENOUS \\
NI & INDIRECT $<$ HETEROGENOUS & - \\
\hline
\end{tabular}

Table 2. Effect of gain on Completion Time (CT), Orientation Error (OE), Orientation Variability (OV) and Number of Inflexion points (NI) for both ballistic (left) and meticulous (right) drawing 
NI than Heterogenous in ballistic drawing. Considering the important distortion of the heterogeneous condition (2:1), our study reveals that the effect of a non homogeneous gain is quite limited.

Taken together, the quantitative and qualitative results indicate that, even though some participants appreciated the reduction in physical movement offered by the gain, departing from a C/D gain of 1 reduced the quality of the production by increasing the variability of the line direction. This is a surprising result considering that the Homogenous condition, for example, required half the amount of pen motion compared to INDIRECT in a similar amount of time, hence participants had less opportunity to make corrections. However, the gain amplified the difficulty of aiming in the correct direction, as revealed in the "direct vs. indirect" analysis, which may explain the greater orientation variability.

We did not include gains lower than 1 in our studies, further studies will be required to study if reducing the gain may improve the quality of the production while remaining at an acceptable level of performance.

\section{CONCLUSION}

In this paper we studied the influence of indirectness on the use of pen devices by novice users in ballistic and meticulous drawing, both in terms of performance and production quality. We showed that indirectness degrades drawing performances and yields greater orientation error and variability depending on the type of gesture. Overall, our experiment indicates that the benefits of the eye-hand dissociation, i.e. removing the hand occlusion and allowing ideal hand and gaze configurations, do not counterbalance the difficulty of inferring the initial orientation of the drawing gesture. In addition, we showed that modifying the gain of the device increases the variability of the stroke orientation.

In the future, we intend to study (1) the evolution of our results in the case of professional users (2) if our results on drawing straight lines generalize to curved lines and (3) how novel interaction techniques could reduce the orientation error and variability when drawing with an indirect pen. In particular, we plan to investigate if a graphical display of the predicted trajectory of the pen can be useful for quickly correcting the drawing gesture.

\section{ACKNOWLEDGMENTS}

This work was partially funded by the French government in the project 3DCI (FUI-AAP14) and the LabEx PERSYVAL-Lab (ANR-11-LABX-0025-01).

\section{BIBLIOGRAPHIE}

1. Accot J. \& Zhai S. Beyond fitts' law: Models for trajectory-based hci tasks. In Proc. CHI'97, ACM (1997), 295-302.

2. Alt H. \& Godau M. Computing the fréchet distance between two polygonal curves. International Journal of Computational Geometry \& Applications 05, 01 n02 (1995), 75-91.

3. Belogay E., Cabrelli C., Molter U. \& Shonkwiler R. Calculating the hausdorff distance between curves. Information Processing Letters 64, 1 (1997), 17 - 22.

4. Bérard F. \& Rochet-Capellan A. The transfer of learning as hci similarity: Towards an objective assessment of the sensory-motor basis of naturalness. In Proc. CHI'15, ACM (2015), 1315-1324.

5. Brenner E., Smeets J. \& Remijnse-Tamerius H. C. M. Curvature in hand movements as a result of visual misjudgements of direction. Spatial Vision 15, 4 (2002), 393-414

6. Deber J., Jota R., Forlines C. \& Wigdor D. How much faster is fast enough?: User perception of latency \& latency improvements in direct and indirect touch. In Proc. CHI'15, ACM (2015), $1827-1836$.

7. Forlines C. \& Balakrishnan R. Evaluating tactile feedback and direct vs. indirect stylus input in pointing and crossing selection tasks. In Proc. CHI'08, ACM (2008), 1563-1572.

8. Gilliot J., Casiez G. \& Roussel N. Impact of form factors and input conditions on absolute indirect-touch pointing tasks. In Proc. CHI'14, ACM (2014), 723-732.

9. Gowen E. \& Miall R. C. Eye-hand interactions in tracing and drawing tasks. Human movement science 25, 4-5 (2006), 568-85.

10. Keefe D., Zeleznik R. \& Laidlaw D. Drawing on air: Input techniques for controlled $3 \mathrm{~d}$ line illustration. Visualization and Computer Graphics 13, 5 (2007), 1067-1081.

11. Ketcham C., Dounskaia N. V. \& Stelmach G. E. The role of vision in the control of continuous multijoint movements. Journal of Motor Behavior 38, 1 (2006), 29-44.

12. Knoedel, Hachet \& Martin. Multi-touch rst in $2 \mathrm{~d}$ and $3 \mathrm{~d}$ spaces: Studying the impact of directness on user performance. In Proc. 3DUI'11, IEE (2011), 75-78.

13. MacKenzie I. S., Kauppinen T. \& Silfverberg M. Accuracy measures for evaluating computer pointing devices. In Proc. CHI'01, ACM (2001), 9-16.

14. Owen R., Kurtenbach G., Fitzmaurice G., Baudel T. \& Buxton B. When it gets more difficult, use both hands: Exploring bimanual curve manipulation. In Proc. GI'05, Canadian Human-Computer Communications Society (2005), 17-24.

15. Reina G. \& Schwartz A. B. Eye-hand coupling during closed-loop drawing: Evidence of shared motor planning? Human Movement Science 22, 2 (2003), 137-152.

16. Schmidt D., Block F. \& Gellersen H. A comparison of direct and indirect multi-touch input for large surfaces. In Proc. INTERACT'09, Springer (2009), 582-594.

17. Shon Y. \& McMains S. Evaluation of drawing on $3 \mathrm{~d}$ surfaces with haptics. Computer Graphics and Applications 24, 6 (2004), 40-50.

18. Tchalenko J. Eye movements in drawing simple lines. Perception 36, 8 (2007), 1152-1167.

19. Vercher J. L., Gauthier G. M., Cole J. \& Blouin J. Role of arm proprioception in calibrating the arm-eye temporal coordination. Neuroscience letters 237, 2-3 (1997), 109-12. 